\title{
VOUCHER Y CIERRE DE ESCUELAS EN CHILE
}

\author{
Ricardo D. Paredes \\ Duoc UC \\ Matías Fresard \\ Duoc UC
}

\begin{abstract}
Resumen: Existe controversia acerca del efecto del sistema de vouchers sobre el aprendizaje, particularmente en el régimen escolar chileno. Por un lado, se arguye que la competencia de las escuelas por capturar y retener más alumnos, con padres que escogen las mejores, generaría un efecto virtuoso en el aprendizaje. Por otro, se dice que los padres no elegirían bien, las escuelas no reaccionarían a la competencia ni el propio Estado limitaría el cierre y la reasignación natural de alumnos. Para contribuir con evidencia en esta discusión, este artículo identifica factores relacionados con el cierre de escuelas en Chile y lo que ocurre con el destino de los alumnos de las escuelas cerradas. Con antecedentes de rendimiento por alumno y funcionamiento de escuelas para el período que va desde el año 2002 hasta el 2012, los resultados sugieren que la cantidad de alumnos por nivel se correlaciona en alto grado y negativamente con la probabilidad de cierre; que hay un quiebre estructural en la probabilidad de cierre al nivel de 42 alumnos por nivel, lo que presumiblemente está determinado por la calidad, y que, frente a la caída de alumnos, las escuelas municipales cierran menos que las escuelas particulares subvencionadas y las particulares pagadas, lo que es consistente con sus mayores restricciones políticas. Finalmente, hallamos que los
\end{abstract}

RicARdo Paredes. Rector de Duoc UC y profesor de la Escuela de Ingeniería de la Pontificia Universidad Católica de Chile (PUC). Email: rparedesm@duoc.cl.

Matías Fresard. Egresado del magíster en análisis económico de la Universidad de Chile. Investigador de la Subdirección de Estudios, Duoc UC. Email: mfresard@, duoc.cl. 
alumnos cuyas escuelas cierran se tienden a mover a otras mejores, lo que desde el punto de vista de la recomposición de la matrícula es una consecuencia virtuosa.

Palabras clave: aprendizaje, cierre de escuelas, sistema de vouchers, escuelas municipales, escuelas subvencionadas.

RECIBIDO: marzo 2018; ACEPTADO: junio 2018.

Clasificación JEL: C24, I21, J24, L25.

\section{VOUCHERS AND SCHOOL CLOSURES IN CHILE}

ABSTRACT: There is controversy about the effect of the voucher system on learning, particularly in the Chilean school system. On the one hand, it is argued that competition between schools to capture and retain more students, with parents choosing the best, should generate a virtuous learning effect. On the other, it is said that parents do not choose well, schools do not react to competition and the State itself limits school closures and the natural reallocation of students. To bring some evidence to the debate, this article identifies factors relating to school closures in Chile and what ultimately happens to the students from the closed schools. Drawing on information about individual student performance and school operation for the period from 2002 to 2012, the results suggest that the number of students per level correlates heavily and negatively with the likelihood of closure; that there is a structural break in the likelihood of closure at the level of 42 students per level, which is presumably determined by quality; and that when the numbers of students drop, municipal schools are less likely to close than subsidized and unsubsidized private schools, which is consistent with the greater political constraints on them. Lastly, we find that students whose schools close tend to move on to better ones, which from the viewpoint of the recomposition of school enrollment is a virtuous consequence.

KEYWORDS: learning, school closure, voucher system, municipal schools, subsidized schools.

RECEIVED: March 2018; ACCEPTED: June 2018.

JEL CLASSIFICATION: C24, I21, J24, L25.

\section{INTRODUCCIÓN}

ara que un sistema de vouchers sea educacionalmente efectivo se requiere, entre otras condiciones, que las escuelas de peor rendimiento pierdan alumnos y eventualmente cierren, mientras que las de mejor rendimiento, los ganen (Beyer 2000). Que las escuelas peores 
cierren tiene un lado negativo para los niños y sus familias, en lo que respecta a los costos de traslado y la pérdida de redes. Por ello, los cierres de escuela son resistidos y están condicionados a factores políticos. Es esperable, así, que la probabilidad de cierre de las escuelas caiga cuando haya menos escuelas alternativas o cuando ellas son públicas.

La discusión de política pública en Chile, uno de los países con el sistema de vouchers más extendido en el mundo, ha dado espacio a un conjunto de medidas que, en la práctica, limitan su funcionamiento. Aedo y Sapelli (2000) revisan un conjunto de trabajos a esa época, remarcando que una de las ventajas más claras del sistema de vouchers es haber aumentado la competencia entre escuelas. Ello habría generado incentivos para dar un mejor servicio educativo, ampliando las posibilidades de elección de las familias de ingresos bajos y medios.

Por su parte, la posición más influyente de la última década sostiene que el sistema de vouchers ha sido negativo para el aprendizaje, porque sobredimensionaría las evaluaciones, exacerbaría la competencia entre alumnos y tendría consecuencias adversas sobre segregación escolar (Corvalán et al. 2015). A partir de lo que ha sido principalmente una hipótesis, en la última década el Estado de Chile ha venido aplicando medidas que van en contra de la esencia del funcionamiento descentralizado de vouchers, tales como el término al copago por parte de los padres, el que se relaciona con la posibilidad de elección; el término del lucro en las escuelas, que limita la diversidad de escuelas, y otras que incluso implican limitaciones a la divulgación de resultados de test de aprendizajes o limitaciones al cierre de las escuelas mal evaluadas. Más aún, el cierre de escuelas se ha considerado como una consecuencia adversa del funcionamiento del sistema de vouchers (ver, por ejemplo, Corvalán et al. 2015), lo que está muy desalineado con un conjunto de estudios que siguieron el pionero trabajo de Aedo y Sapelli (2000).

Parte importante de la discusión en países que tienen sistemas de vouchers restringidos también se relaciona con el cierre de escuelas, particularmente si no son capaces de cumplir estándares de calidad (Chumacero y Paredes, 2012). En Chile, ello se ha mezclado con un problema de sustentabilidad financiera, sustentabilidad que por cierto es más precaria en un régimen de vouchers y resulta particularmente crítica para las escuelas que pierden alumnos a causa de su baja calidad. 
En este trabajo dimensionamos la magnitud del cierre de escuelas en Chile e identificamos los factores que se relacionan con la probabilidad de cierre. Para ello, utilizamos una metodología que contempla la posibilidad de un quiebre estructural en base al tamaño de la escuela, lo que le da flexibilidad al modelo y evita, de existir tal quiebre, sesgos de estimación. Hallamos que existe un quiebre estructural al nivel de 42 alumnos por nivel, pasado el cual la probabilidad de cierre cae significativamente. Hallamos también que, condicional al tamaño, no se aprecia una relación estable entre la probabilidad de cierre y el rendimiento de la escuela. Sin embargo, y más relevante respecto de las prescripciones de política, encontramos que los alumnos de las escuelas que cierran migran mayoritariamente a mejores escuelas.

Más allá de esta introducción, el resto del trabajo se estructura en tres secciones. La sección 2 entrega los elementos centrales del funcionamiento del sistema educacional en Chile y hace una breve revisión de la literatura sobre vouchers. La sección 3 describe los datos, presenta la metodología para la estimación sobre cierre de escuelas y reporta los resultados. La sección 4 concluye.

\section{ANTECEDENTES}

\subsection{El sistema de vouchers en Chile}

La reforma del sistema educativo chileno, de principios de los ochenta, generó básicamente tres categorías de escuela, de acuerdo a su financiamiento. En primer lugar, las escuelas municipales. En segundo lugar, las particulares subvencionadas. Estas dos son financiadas por el Estado vía vouchers, que se determinan de acuerdo al nivel de asistencia promedio de cada alumno durante los últimos tres meses. Y en tercer lugar, se definieron los colegios particulares pagados, los que no reciben aportes del Estado y se financian únicamente a través de matrículas, mensualidades y donaciones de terceros. Estos tres tipos de escuela tienen diferencias significativas, incluyendo la distinta administración. Las escuelas municipales son administradas por la municipalidad o corporaciones municipales; los colegios particulares subvencionados y los particulares pagados, por instituciones que pueden tener o no fines de lucro (Aedo y Sapelli 2000). También aplican a cada cual distintos 
regímenes laborales, siendo más difícil el despido en las escuelas municipales. Junto con esto, a las escuelas municipales - con mínimas excepciones - se les impidió, hasta el año 2017, seleccionar a sus alumnos, a menos de que se vean enfrentadas a un exceso de demanda. Esta diferenciación ha generado controversias sobre los efectos en segregación y aprendizaje, las que han derivado en nuevas regulaciones, las que incluso contemplan el término del sistema de vouchers (Paredes et al. 2013; Zubizarreta et al. 2014).

\subsection{La literatura}

La literatura sobre las consecuencias del sistema de vouchers en el aprendizaje es amplia. En el plano internacional, Levin (1998) y Neal (2002) analizan varias experiencias y concluyen con una visión crítica sobre su efecto en el aprendizaje, que atribuyen a la incapacidad del sistema de disciplinar a las escuelas, porque las personas no procesarían bien la información, lo que sería en un alto grado en las de menor ingreso y educación. En la misma línea, Kisida y Wolf (2010), Hastings y Weinstein (2008), Dronkers y Avram (2015) sugieren que la falta de información limita la operación de un sistema de vouchers. Así, Hastings et al. (2007) muestran que la entrega de información impacta de manera positiva en el rendimiento académico de la escuela escogida.

Para Chile, Carnoy y McEwan (2001), McEwan y Carnoy (2000) y Elacqua y Fabrega (2004) atribuyen los pobres resultados en aprendizaje en el sistema completo a la baja capacidad de los padres de elegir escuelas de mejor calidad. Ello es, en parte, apoyado por encuestas de conocimiento del Simce (ver, por ejemplo, CEP 2006), que muestran que menos del 50 por ciento de los padres conoce los resultados de los test estandarizados, y menos del 1 por ciento considera esos resultados como el factor más incidente a la hora de elegir una escuela. Sin embargo, Gallego et al. (2008) destacan que la elección de los padres pone énfasis en calidad y distancia, creciendo la importancia de la primera y disminuyendo la segunda a medida que aumenta el ingreso familiar. Chumacero et al. (2011) confirman esos hallazgos a través de un modelo de elección de escuela más cercana y concluyen que la distancia y la calidad son los atributos más relevantes en la elección de la escuela. Gómez et al. (2012) analizan el efecto que tuvo sobre la decisión de los 
padres el informar públicamente los resultados por escuela. Hallan un efecto positivo de la información, pero destacan que, aun en el tiempo cuando dicha información no era pública, los padres también actuaban "como si" supieran sobre diferenciales de calidad.

Más recientemente, Arteaga et al. (2018) desarrollan un modelo de elección de escuela restringiendo el conjunto de preferencias relevantes y usando directamente la georreferenciación de establecimientos y hogares. Aunque centran su foco en el efecto de la segregación del sistema de copago, confirman que la distancia, el pago y la calidad son factores relevantes en la elección de escuelas por parte de los padres. Concluyen, además, que el factor que más incide en la segregación escolar - una de las consecuencias atribuidas al sistema de vouchers por varios autores - no es la capacidad de pago, sino el currículo de las escuelas.

En resumen, la literatura es relativamente crítica de la capacidad de los padres para elegir escuela; crítica, al menos, en señalar que dicha elección es acotada y mediada por el ingreso familiar. Para el caso de Chile, la literatura más reciente halla un efecto claro de la calidad en la elección de escuelas, sin perjuicio de la capacidad diferenciada, entre familias, de procesar la información y definir acorde a ella. De cualquier modo, incluso los hallazgos más recientes, en los que se observa que la elección de escuela está en parte determinada por la calidad, no dan luces sobre cuánta información es necesaria para que observemos un efecto positivo en los aprendizajes. Podría ser suficiente que un pequeño porcentaje de los padres reaccione a las diferencias de calidad, de tal suerte que pueda provocar efectos significativos en la sustentabilidad financiera de las escuelas. Así, si producto de una baja marginal de estudiantes se gatillara una crisis financiera y el cierre de la escuela, ello podría tener un efecto inmediato y significativo. Por el contrario, si la salida de alumnos deteriora la capacidad de proveer calidad pero ello no involucra un cierre, una baja relevancia de la calidad de la escuela en la decisión de los padres podría potenciar los efectos adversos sobre todo el sistema.

Una línea de literatura que sortea la pregunta respecto a cuál es el nivel límite de información necesaria para que existan efectos significativos en el aprendizaje se enfoca directamente en el efecto de la competencia entre escuelas. Borland y Howsen (1992) y Couch et al. (1993), pioneros al considerar distintas medidas de competencia, veri- 
fican una relación positiva entre ellas y el resultado académico. Hoxby (1994; 2001) halla que la mayor competencia, provista por las escuelas privadas, aumenta la eficiencia de las públicas. Sander (1999), usando una metodología de estimación algo más completa, encuentra que la competencia generada por escuelas privadas no impacta el resultado de las públicas. Ladd y Fiske (2001), para Nueva Zelanda, hallan que las condiciones competitivas provocadas por la reforma en ese país redujeron el aprendizaje. Böhlmark y Lindahl (2008) estudian la competencia entre escuelas privadas y públicas al interior de la comunas en Suecia después de creado un sistema de vouchers. Encuentran que un aumento en la participación de las escuelas privadas mejora los resultados de las escuelas públicas a corto plazo, pero no dan con evidencia para el mediano y largo plazo, concluyendo que el efecto de esta competencia es discreto en producir efectos positivos y duraderos.

Para Chile, McEwan y Carnoy (2000), también utilizando datos de corte transversal, analizan el efecto de la competencia - medida como porcentaje de la matrícula de escuelas particulares en la comuna- en el rendimiento de las escuelas para cuarto básico entre 1988 y 1996. Sus resultados sugieren un efecto negativo en las escuelas municipalizadas y que este efecto es mayor en aquellas comunas con una mayor participación de escuelas privadas subvencionadas. Hsieh y Urquiola (2003) hallan que el aprendizaje disminuye a medida que aumenta la presencia de escuelas privadas subvencionadas en la comuna, resultado que se replica con otras variables dependientes utilizadas para el mismo estudio (tasa de repitencia, promedio de escolaridad de los alumnos). Gallego (2002) utiliza como proxy de la variable competencia el porcentaje de matricula privada a nivel comunal, y como instrumentos, la proporción de población urbana y la cantidad de estudiantes matriculados por comuna. Observa una relación positiva entre competencia y resultados educativos sólo para las escuelas privadas subvencionadas, y destaca que la diferencia con los resultados previos puede obedecer a no considerar la posible endogeneidad de la variable competencia.

En un trabajo más reciente, Gallegos et al. (2016) critican las medidas de competencia utilizadas en la literatura que emplea datos de corte transversal, sugiriendo que sesgan hacia abajo cualquier efecto de la competencia en el aprendizaje. Si la decisión de localizar una escuela es libre, debiera terminar igualándose transversalmente los aprendiza- 
jes. La forma en que proponen estudiar el efecto de la competencia es usar una variable diferente, aprovechando un cambio institucional que efectivamente permite observar el efecto de la competencia. Los resultados sugieren que una mayor competencia aumenta significativamente el rendimiento educacional de escuelas tanto privadas como municipales. El efecto, sin embargo, resulta sustancialmente mayor en el caso de las escuelas privadas, lo que lo atribuyen a la mayor flexibilidad e incentivos directos que hay para las escuelas privadas de tener una mejor gestión financiera.

Sobre el cierre de escuelas, hay, sustancialmente, menos trabajos; además, ellos se centran en elementos sociológicos, ya que el cierre es una decisión que excede ampliamente lo económico. Phipps (1993) analiza el efecto de distintas interacciones entre la comunidad y la directiva de la escuela respecto a las decisiones de cierre en los Estados Unidos. Destaca los costos asociados a clausurar una escuela, por la pérdida de un bien público usado por toda la comunidad, el cambio en el estándar de vida de esa comunidad, los mayores requerimientos de viaje, y el efecto adverso en la identidad local. Concluye que los representantes de la comunidad se tienden a involucrar, a un mayor grado, sólo en el caso de que la vida de toda la comunidad estuviera relacionada con la escuela, y que los miembros de la directiva intentan usar sus poderes y capacidades para disminuir lo más posible los costos asociados al cierre.

Pandey et al. (2009) sugieren que la probabilidad de cierre depende de ciertas características de cada escuela, tales como la razón alumnoprofesor, la cantidad de profesores, el salario de los mismos, el porcentaje de alumnos negros y católicos, si se trata de fundaciones sin fines de lucro, y el tamaño. Hallan que las escuelas más nuevas, de menor tamaño, tienen menor probabilidad de cierre y que el hecho de ser escuelas religiosas o "high-school-only" no son factores estadísticamente significativos en diferenciar su probabilidad de cierre.

Billger y Beck (2009) analizan escuelas rurales en Illinois. Hallan que la cantidad de alumnos promedio para las escuelas secundarias que no cerraron era de 689 , más del triple del promedio de las que sí cerraron (que era de 202). Al mismo tiempo, las escuelas que cesaron sus actividades tendían a encontrarse en las comunidades con menores niveles de ingresos. Sus resultados muestran que la cantidad de alumnos 
está correlacionada negativamente con la probabilidad de cierre y que el que la escuela se encuentre en una zona rural aumenta el riesgo de cierre. Billger (2010) postula diferentes razones por las cuales una escuela decide cerrar. Enfocándose principalmente en escuelas primarias (elementary school) y secundarias (high school) de Illinois y usando probit, plantea una prociclicidad del cierre de escuelas, y que este cierre aumenta con la proporción de alumnos negros y de bajos recursos. Sugiere que, condicional a la tasa de pobreza, empleabilidad agrícola, población inmigrante, logros escolares, población educada y tasa de desempleo, la baja cantidad de alumnos y el aumento en costos no necesariamente se relacionan con el cierre.

Para Chile, Grau et al. (2015) sugieren que los cierres de escuelas inciden en la probabilidad de que el alumno baje su rendimiento en la nueva escuela a la que debe asistir. El cierre de escuela aumenta en 30 por ciento (1,5 puntos porcentuales) la probabilidad de que el alumno repita en la nueva institución.

\section{DATOS Y METODOLOGÍA}

\subsection{Cierre de escuelas}

La base de datos empleada tiene un formato panel y corresponde a una unión entre las bases Simce, test de aprendizaje estandarizado y bases del Mineduc, la cual tiene información sobre todas las escuelas existentes. El período considerado es 2002 a 2013, lo que permite un seguimiento de los alumnos por un tiempo significativo. Las bases Simce contienen los puntajes de los test y características sociodemográficas de los alumnos y de sus familias. Así, disponemos de información de escuelas, rendimiento de cada alumno y de sus características sociodemográficos, incluyendo el nivel de ingresos de los padres.

La base de escuelas permite, a través de un código identificador (RBD), seguirlas a través del tiempo. Utilizamos un conteo de dos etapas, con el fin de identificar las escuelas que han cerrado en el período estudiado. Para la primera etapa, utilizamos el RBD; si este código "desaparece", por más de dos años, entonces marcamos la escuela como potencialmente cerrada. Con el propósito de eliminar errores de cambio o errores de RBD, la segunda etapa considera todas las escuelas 
potencialmente cerradas. Para cada una de ellas, calculamos el mayor porcentaje de alumnos que se cambiaron a una escuela con un código diferente. Si más del 80 por ciento de los alumnos de la escuela supuestamente cerrada aparece bajo una misma escuela pero con otro código, asumimos que esa escuela no cerró, sino que sólo cambió su código. $\mathrm{Si}$ el máximo porcentaje de alumnos que aparecen, bajo otro código, es inferior a 80 por ciento, entonces asumimos que efectivamente la escuela cerró.

Del conteo de escuelas cerradas (tabla 1), se aprecia que en relación al total de escuelas de su tipo, los colegios particulares pagados tienen un porcentaje de cierre sustancialmente mayor al de las escuelas privadas subvencionadas y municipales. ${ }^{1}$

Tabla 1. ESCUELAS CERRADAS POR DEPENDENCIA

\begin{tabular}{|c|c|c|c|c|c|c|c|}
\hline \multirow[b]{3}{*}{ Año de cierre } & \multicolumn{6}{|c|}{ Tipo de dependencia } & \multirow[b]{3}{*}{ Total } \\
\hline & \multicolumn{2}{|c|}{ Municipal } & \multicolumn{2}{|c|}{$\begin{array}{c}\text { Particular } \\
\text { subvencionado }\end{array}$} & \multicolumn{2}{|c|}{ Particular pagado } & \\
\hline & Cerrados & $\%$ & Cerrados & $\%$ & Cerrados & $\%$ & \\
\hline 2002 & 108 & 1,88 & 27 & 0,92 & 51 & 8,46 & 186 \\
\hline 2003 & 94 & 1,65 & 46 & 1,46 & 43 & 7,29 & 183 \\
\hline 2004 & 123 & 2,17 & 40 & 1,24 & 20 & 3,58 & 183 \\
\hline 2005 & 184 & 3,25 & 28 & 0,83 & 11 & 2,39 & 223 \\
\hline 2006 & 109 & 1,97 & 43 & 1,23 & 6 & 1,34 & 158 \\
\hline 2007 & 112 & 2,04 & 55 & 1,56 & 17 & 3,79 & 184 \\
\hline 2008 & 81 & 1,50 & 57 & 1,59 & 7 & 1,56 & 145 \\
\hline 2009 & 159 & 2,95 & 41 & 1,13 & 7 & 1,59 & 207 \\
\hline 2010 & 200 & 3,78 & 49 & 1,34 & 11 & 2,47 & 260 \\
\hline 2011 & 149 & 2,89 & 112 & 3,04 & 10 & 2,29 & 271 \\
\hline 2012 & 94 & 1,84 & 73 & 1,97 & 11 & 2,59 & 178 \\
\hline Total & 1.413 & & 571 & & 194 & & 2.178 \\
\hline
\end{tabular}

${ }^{1}$ En la tabla A1 del Anexo se muestra las escuelas que abrieron en cada año, para lo que se utilizó el mismo método descrito para contabilizar el cierre. La mayor cantidad de escuelas que entraron, tanto en términos absolutos como relativos, son particulares subvencionadas, lo que refleja un cambio estructural descrito en varios trabajos (por ejemplo, Paredes y Pinto 2009). 
Por su parte, como se observa en la tabla 2, de las escuelas que cerraron la gran mayoría poseía menos de 10 alumnos por nivel el año de cierre. Esa tabla indica, también, que las escuelas que cerraron están sobrerrepresentadas en escuelas de bajo puntaje Simce.

Tabla 2. CIERRE POR TAMAÑO Y RESULTADOS ACADÉMICOS

\begin{tabular}{|c|c|c|c|c|c|c|c|}
\hline Tamaño & $1-10$ & $11-20$ & $21-30$ & $31-40$ & $41-50$ & 51 o más & \\
\hline $\begin{array}{l}\text { Total de } \\
\text { escuelas }\end{array}$ & $41,78 \%$ & $10,92 \%$ & $8,98 \%$ & $7,36 \%$ & $4,91 \%$ & $26,05 \%$ & \\
\hline $\begin{array}{l}\text { Escuelas } \\
\text { cerradas }\end{array}$ & $75,21 \%$ & $7,94 \%$ & $4,77 \%$ & $3,35 \%$ & $2,11 \%$ & $6,06 \%$ & \\
\hline Simce & $1-100$ & $101-150$ & $151-200$ & $201-250$ & $251-300$ & $301-350$ & $351-400$ \\
\hline \multicolumn{8}{|c|}{ Total } \\
\hline Lenguaje & $0,017 \%$ & $0,086 \%$ & $2,745 \%$ & $62,61 \%$ & $29,80 \%$ & $4,726 \%$ & $0,017 \%$ \\
\hline Matemáticas & $0,035 \%$ & $0,195 \%$ & 3,762\% & $65,72 \%$ & $26,01 \%$ & $4,027 \%$ & $0,248 \%$ \\
\hline \multicolumn{8}{|c|}{ Cerradas } \\
\hline Lenguaje & $0,152 \%$ & $0,610 \%$ & $9,146 \%$ & $51,83 \%$ & $31,40 \%$ & $6,707 \%$ & $0,152 \%$ \\
\hline Matemáticas & $0,308 \%$ & $1,541 \%$ & $12,33 \%$ & $53,78 \%$ & $25,27 \%$ & 6,626\% & $0,154 \%$ \\
\hline
\end{tabular}

La descripción previa sugiere que las peores escuelas pierden alumnos y cierran. Sin embargo, pudiera ser que las escuelas con menos alumnos sean menos sustentables financieramente; o sea, que su cierre no tenga que ver con la calidad, sino con el aislamiento geográfico, con la ruralidad. En tal caso, la supervivencia de la escuela debiera ser cuidada. Naturalmente, calidad y número de alumnos se relacionan bajo un esquema de vouchers, por lo que estimaciones condicionadas son de utilidad y es lo que hacemos a continuación.

\subsection{Factores asociados al cierre de escuelas}

Un factor de preocupación, que es esperable que esté detrás del cierre de las escuelas, es la calidad. La relación de la calidad con el cierre, en un sistema de vouchers, se debiera dar a través de la caída de estudiantes. Así, la vinculación empírica más directa es entre cierre y número de estudiantes, lo que determina el tamaño. Por su parte, la relación entre tamaño y cierre puede no ser lineal. La decisión de cierre debiera tener que ver con un umbral bajo el cual no es financieramente sostenible la mantención de una escuela. 
Las estimaciones de relaciones condicionadas tienen el potencial de aislar los efectos de distintos factores, de forma que podemos identificar empíricamente la importancia de cada uno de ellos. La ausencia de una teoría asentada y el propósito de investigar sobre dichas correlaciones nos lleva a considerar un modelo probit simple, que considera, entre otras, las variables calidad (medida a través de los resultados medios de los test estandarizados), matrícula por nivel, dependencia e ingreso familiar de los alumnos. La variable dependiente cierre corresponde a una dummy que indica si la escuela cerró en el periodo de estudio.

Una preocupación particular por esta estimación es la posibilidad, ciertamente derivada de la lógica financiera, de que exista un quiebre de matrícula bajo el cual la viabilidad financiera de una escuela (que fundamentalmente depende de los ingresos vía voucher) cae fuertemente. En simple, se espera que la relación entre tamaño y probabilidad de cierre sea no lineal y que, de presentar un quiebre, se requiere un método de estimación que contemple esa posibilidad.

Como en este caso no conocemos el eventual punto de quiebre, requerimos una metodología, en la línea de la propuesta por Hansen (2000) para modelos lineales o la propuesta por Wongsosaputro et al. (2011), ya que eliminan el posible sesgo en la estimación, lo que se exacerba en el caso de la estimación probit. Específicamente, este modelo estimado, considerando la estructura de panel, considera la estimación probit para dos submuestras:

$$
\begin{aligned}
& y_{i}=\left\{\begin{array}{c}
1 \text { si } y_{i}^{*}<0 \\
0 \text { caso contrario }
\end{array}, i=1, \ldots, \pi N\right. \\
& y_{i}=\left\{\begin{array}{c}
1 \text { si } y_{i}^{*}<0 \\
0 \text { caso contrario }
\end{array}, i=(\pi N+1), \ldots, N\right.
\end{aligned}
$$

En donde $\pi \in[0,1]$ corresponde al porcentaje de la muestra antes del punto de quiebre, que es desconocido, y $\pi \mathrm{N}$ representa el punto del quiebre. Una manera de testear los posibles puntos de quiebre es utilizar una expresión modificada del test Log-likelihood Ratio (LR), como:

$$
L R_{N}(\pi)_{\pi \in \Pi}=2\left[l_{1}\left(\widehat{\beta_{1}}\right)+l_{2}\left(\widehat{\beta_{2}}\right)-l(\hat{\beta})\right]
$$


Donde $l_{1}($.$) y l_{2}($.$) corresponden a las funciones log-likelihood de la$ primera y segunda estimación, respectivamente.

Nos centraremos en el test LR, ya que sólo se necesita del modelo restringido para calcularlo. Para ello estimamos el modelo probit asumiendo la hipótesis nula de que no existe un quiebre estructural en la estimación, con lo cual se obtiene la probabilidad estimada de cierre para cada escuela y los residuos de la estimación. Luego, se realizan dos estimaciones, según las observaciones bajo y sobre el porcentaje $(\pi)$ de la muestra que se está evaluando.

Los resultados del test de quiebre estructural, que siguen el método de Andrews, indican que los datos son consistentes con la existencia de éste, que corresponde a 42 alumnos por nivel y que, superando ese nivel, la probabilidad de cierre cae drásticamente. ${ }^{2}$ Los resultados de las estimaciones, para los dos regímenes, antes y después del quiebre, se presentan en la tabla 3 .

Figura 1. ESTIMACIÓN DEL QUIEBRE ESTRUCTURAL EN UN MODELO PROBIT CANTIDAD DE ALUMNOS POR NIVEL

Muestra completa de colegios

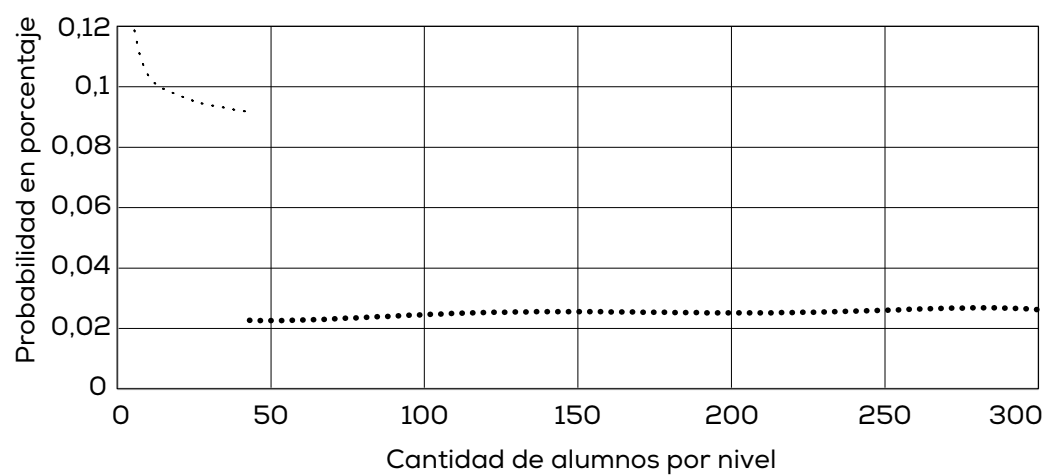

. - - Prob. antes del quiebre ........Prob. después del quiebre

La importancia del quiebre estructural se hace especialmente visible en la figura 1. El hecho de que el quiebre se produzca al nivel de 42 alumnos por nivel (puntaje que acumula algo más del 50 por ciento de

${ }^{2}$ Los valores de este test son obtenidos de Andrews (1993) (tablas 11 y 12), para $\Pi=[0,01 ; 0,99]$. 
Tabla 3. EFECTO FIJO RESPECTO AL CIERRE DE ESCUELA ANTES Y DESPUÉS DEL QUIEBRE ESTRUCTURAL

\begin{tabular}{|c|c|c|}
\hline & $\begin{array}{c}\text { Menos de } 42 \\
\text { alumnos por nivel }\end{array}$ & $\begin{array}{c}42 \text { o más } \\
\text { alumnos por nivel }\end{array}$ \\
\hline \multirow[t]{2}{*}{ Matrícula por nivel } & $-0,000535$ & $0,0003579^{* * *}$ \\
\hline & $(0,00064)$ & $(0,00008)$ \\
\hline \multirow[t]{2}{*}{ Matrícula por nivel ${ }^{2}$} & 0,00000467 & $-0,00000059^{* * *}$ \\
\hline & $(0,00000)$ & $(0,0000000)$ \\
\hline \multirow[t]{2}{*}{ Escuela subvencionada } & 0,0249464 & 0,0126581 \\
\hline & $(0,11993)$ & $(, 05006)$ \\
\hline \multirow[t]{2}{*}{ Colegio particular pagado } & $-0,0323318$ & $-0,0794297$ \\
\hline & $(0,12175)$ & $(0,05649)$ \\
\hline \multirow[t]{2}{*}{ Matrícula por nivel / Escuela subvencionada } & $-0,0001799$ & 0,000063 \\
\hline & $(0,00051)$ & $(0,00008)$ \\
\hline \multirow[t]{2}{*}{ Matrícula por nivel / Colegio p. pagado } & 0,0010571 & $0,0007125^{*}$ \\
\hline & $(0,00121)$ & $(0,00033)$ \\
\hline \multirow[t]{2}{*}{ Puntaje Simce promedio de matemáticas } & 0,00032 & $-0,0002081$ \\
\hline & $(0,00084)$ & $(0,00045)$ \\
\hline \multirow[t]{2}{*}{ Puntaje Simce promedio de matemáticas ${ }^{2}$} & $-0,00000227$ & 0,000000318 \\
\hline & $(0,00000)$ & $(0,000000)$ \\
\hline \multirow[t]{2}{*}{ Puntaje Simce promedio de lenguaje } & 0,0011471 & 0,0005247 \\
\hline & $(0,00084)$ & $(0,00046)$ \\
\hline \multirow[t]{2}{*}{ Puntaje Simce promedio de lenguaje ${ }^{2}$} & $-0,00000257$ & $-0,00000135$ \\
\hline & $(0,00000)$ & $(0,00000)$ \\
\hline \multirow[t]{2}{*}{ Ingreso familiar promedio } & $0,000000232^{* * *}$ & $6,46 e-8^{* * *}$ \\
\hline & $(0,000000)$ & $(0,000000)$ \\
\hline \multirow[t]{2}{*}{ Matrícula por nivel (urbano) } & $0,00355^{* * *}$ & 0,0001876 \\
\hline & $(0,00188)$ & $(0,00037)$ \\
\hline \multirow[t]{2}{*}{ Constante } & $-0,45088$ & $-0,8970294^{*}$ \\
\hline & $(0,3605722)$ & $(0,3905985)$ \\
\hline Control por efecto fijo (año) & Si & Si \\
\hline Observaciones & 15192 & 24578 \\
\hline Pseudo $R^{2}$ & 0,0595 & 0,0387 \\
\hline$\chi^{2}$ & 391,92 & 165,22 \\
\hline
\end{tabular}

Errores estándar en paréntesis; ${ }^{\star} p<0,1,{ }^{* \star} p<0,05,{ }^{\star \star *} p<0,01$. 
las escuelas) muestra, en primer lugar, que hasta ese puntaje los alumnos adicionales reducen la probabilidad de cierre. Más importante, pasado un tamaño, por así decirlo, "crítico", la probabilidad de cierre cae significativamente.

En cuanto al resto de las variables consideradas, ni el puntaje Simce en lenguaje ni en matemáticas presentan significancia estadística. Esto sugiere que la calidad de la escuela no parece afectar directamente la decisión de cierre, aunque ella sí pudiera hacerlo por la vía de la reducción de la matrícula. De hecho, una estimación que excluye la variable "matrícula" por nivel, lineal y al cuadrado, muestra una correlación condicional negativa entre el puntaje Simce de lenguaje y la probabilidad de cierre.

Por su parte, los coeficientes de las variables interactivas, entre matrícula y colegio particular y escuela particular subvencionada, son positivas y significativas, reflejando que las escuelas municipales (base) responden sustancialmente menos con cierres a las caídas en su matrícula. Ello es consistente con la idea según la cual, detrás de la decisión de cierre, el factor político es sustancialmente mayor en el caso de las escuelas públicas. En particular, la resistencia de los padres al cierre induciría más a los encargados de las escuelas públicas a postergar la decisión de cierre.

Una especificación diferente podría, en cierta forma, abordar el hecho de que el tamaño de la escuela quizá está determinado por la capacidad de recibir alumnos. Estimamos así la misma especificación previa, pero ahora considerando la tasa de desocupación de las escuelas en remplazo de la matrícula por nivel. La tasa de desocupación refleja el déficit de estudiantes respecto de lo planificado. Los resultados confirman la existencia del quiebre en el mismo sentido que lo hace el tamaño. En efecto, en la figura 2 se muestra que el punto de quiebre se da cuando la capacidad no utilizada supera el 49 por ciento. ${ }^{3}$

${ }^{3}$ La cantidad máxima de matrículas para cada colegio se definió como la cantidad máxima de alumnos que presenta cada colegio a lo largo del periodo de estudio más el 10 por ciento de dicha cantidad. 
Figura 2. ESTIMACIÓN DEL QUIEBRE ESTRUCTURAL EN UN MODELO PROBIT. TASA DE DESOCUPACIÓN DE COLEGIO

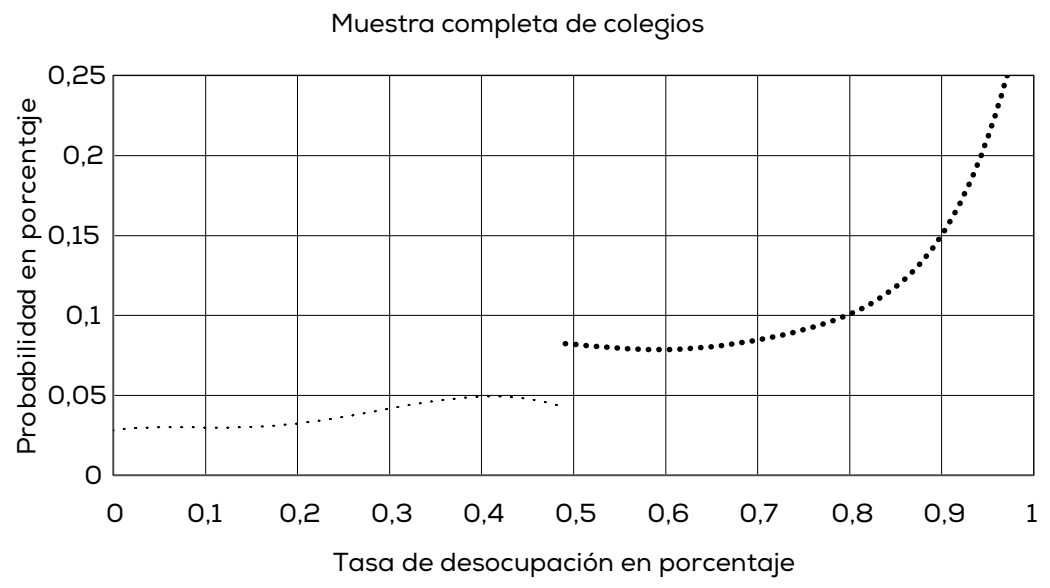

Prob. antes del quiebre

Prob. después del quiebre

\section{3. ¿Qué ocurre con los alumnos desplazados?}

Una pregunta esencial, respecto del efecto que tiene el cierre de escuelas sobre la potencialidad de incrementar el aprendizaje, es ver qué pasa con los alumnos desplazados, lo que se relaciona con cuán buenas son las escuelas que cierran comparadas con aquellas que los reciben. Para aislar los efectos de la composición sociodemográfica de las escuelas, tomamos los residuos de una típica regresión de rendimiento (véase Drago y Paredes 2011) y que estimada para el período 2002-2010 considera, como determinantes del puntaje Simce, el ingreso familiar, la educación de los padres y el haber pasado por educación preescolar, entre otras. De esta regresión excluimos todas las variables de la escuela, con lo que los residuos entregan una proxy del aprendizaje en la escuela que no está explicado por el background socioeconómico.

Con los residuos determinamos un ranking de todas las escuelas de nuestra muestra, y de esta manera determinamos si el cambio de escuela es o no positivo en términos de exponer a los alumnos a un ambiente de mayor desafío y aprendizaje. Los resultados de la tabla 4 muestran que la gran mayoría de los alumnos, al cambiarse porque la escuela cierra - y para todos los años-, lo hace a una mejor escuela. 
Tabla 4. MEJORAS ASOCIADAS AL TRASLADO DESDE ESCUELAS CERRADAS

\begin{tabular}{|c|c|c|c|c|c|}
\hline & & \multicolumn{2}{|c|}{ Simce lenguaje } & \multicolumn{2}{|c|}{ Simce matemáticas } \\
\hline & & Mejor & Peor o igual & Mejor & Peor o igual \\
\hline \multirow{2}{*}{ 2002-2003 } & Cantidad & 14.670 & 5.044 & 14.772 & 4.942 \\
\hline & Porcentaje & $74,41 \%$ & $25,59 \%$ & $74,93 \%$ & $25,07 \%$ \\
\hline \multirow{2}{*}{ 2003-2004 } & Cantidad & 7.926 & 4.167 & 7.513 & 4.580 \\
\hline & Porcentaje & $65,54 \%$ & $34,46 \%$ & $62,13 \%$ & $37,87 \%$ \\
\hline \multirow{2}{*}{ 2004-2005 } & Cantidad & 7.771 & 2.359 & 7.682 & 2.448 \\
\hline & Porcentaje & $76,71 \%$ & $23,29 \%$ & $75,83 \%$ & $24,17 \%$ \\
\hline \multirow{2}{*}{ 2005-2006 } & Cantidad & 8.966 & 2.953 & 9.304 & 2.615 \\
\hline & Porcentaje & $75,22 \%$ & $24,78 \%$ & $78,06 \%$ & $21,94 \%$ \\
\hline \multirow{2}{*}{ 2006-2007 } & Cantidad & 8.538 & 1.664 & 8.596 & 1.606 \\
\hline & Porcentaje & $83,69 \%$ & $16,31 \%$ & $84,26 \%$ & $15,74 \%$ \\
\hline \multirow{2}{*}{ 2007-2008 } & Cantidad & 7.705 & 3.687 & 8.054 & 3.338 \\
\hline & Porcentaje & $67,64 \%$ & $32,36 \%$ & 70,7 & 29,3 \\
\hline \multirow{2}{*}{ 2008-2009 } & Cantidad & 5.890 & 1.095 & 6.055 & 930 \\
\hline & Porcentaje & $84,32 \%$ & $15,68 \%$ & 86,69 & 13,31 \\
\hline \multirow{2}{*}{ 2009-2010 } & Cantidad & 5.468 & 4.355 & 6.942 & 2.881 \\
\hline & Porcentaje & $55,67 \%$ & $44,33 \%$ & $70,67 \%$ & $29,33 \%$ \\
\hline
\end{tabular}

\section{CONCLUSIONES}

Los resultados de la experiencia chilena muestran, en primer lugar, que el cierre de escuelas difiere según su tipo, siendo las escuelas públicas sustancialmente menos sensibles a cerrar debido a un bajo número de alumnos. Ello es consistente con distintas hipótesis que no han sido el propósito de explorar aquí, entre las que se incluye la sobrerrepresentación de escuelas públicas en zonas aisladas - y, por lo tanto, la necesidad social de mantenerlas abiertas_-, así como la existencia de motivaciones estrictamente políticas, que impide tomar decisiones dolorosas pero necesarias, y que comprometen la educación de generaciones.

En segundo lugar, los datos muestran que, a diferencia de lo afirmado por Billger (2010), el tamaño de la escuela es un factor determinante en su cierre. Esta diferencia en las conclusiones puede obedecer a que, en el caso de Estados Unidos, los alumnos tienden a asistir a la escuela más cercana a sus hogares y no se espera que la elección de los padres determine la viabilidad financiera de la escuela. 
En tercer lugar, hallamos que existe un quiebre estructural en el cierre de escuelas, y que ese quiebre se representa en un número crítico de alumnos bajo el cual la pérdida adicional de alumnos reduce fuertemente la viabilidad de los establecimientos.

Finalmente, hallamos una recomposición positiva del cierre de escuelas. Las que se cierran obligan a una reubicación de sus estudiantes, los que en general terminan haciéndolo en escuelas en las que reciben un mayor aprendizaje, y, presumiblemente, tienen mayor exigencia. A su vez, ello podría explicar aumentos de fracaso escolar generados por el cambio de escuela que han sido reportados en la literatura. No obstante, esto sería consistente con un efecto positivo del cierre de escuelas sobre el sistema escolar.

\section{REFERENCIAS}

Aedo, C., \& C. Sapelli. 2000. "El sistema de vouchers en la educación: una revisión de la teoría y la evidencia empírica para Chile". Punto de Referencia 307, Centro de Estudios Públicos.

Andrews, D. W. 1993. "Tests for Parameter Instability and Structural Change with Unknown Change Point”. Econometrica 61 (4): 821-856.

Arteaga, F., V. Paredes \& R. Paredes. 2018. "School Segregation in Chile: Residence, Co-payment, or Preferences". Documento de Trabajo, Departamento de Ingeniería Industrial, PUC.

Beyer, H. 2000. "Entre la autonomía y la intervención: las reformas de la educación en Chile". En La transformación económica de Chile, 643-709. Santiago: Centro de Estudios Públicos.

Billger, S. M. 2010. "Demographics, Fiscal Health, and School Quality: Shedding Light on School Closure Decisions". IZA Discussion Papers 4739.

Billger, S. \& F. D. Beck. 2009. "The Determinants of High School Closures: Lessons from Longitudinal Data throughout Illinois". IZA Discussion Papers 4641.

Böhlmark, A. \& M. Lindahl. 2008. "Does School Privatization Improve Educational Achievement? Evidence from Sweden's Voucher Reform”. IZA Working Paper 3691.

Borland, M. V. \& R. M. Howsen. 1992. "Student Academic Achievement and the Degree of Market Concentration in Education". Economics of Education Review 11 (1): 31-39.

Carnoy, M. \& P. J. McEwan. 2001. "Privatization through Vouchers in Developing Countries: The Cases of Chile and Colombia". En Privatizing education, editado por H. M. Levin, 151-177. Boulder: Westview Press.

CEP. 2006. Evaluación de la Calidad de la Educación en Chile. Parte I. En Encuesta Nacional de Opinión Pública. CEP. 
Chumacero, R. A., D. Gómez \& R. D. Paredes. 2011. "I Would Walk 500 Miles (If It Paid): Vouchers and School Choice in Chile". Economics of Education Review 30 (5): 1103-1114.

Chumacero, R. A. \& R. D. Paredes. 2012. "Vouchers, Choice, and Public Policy: An Overview". Estudios de Economía 39 (2).

Corvalán, J., A. Carrasco \& J. E. García-Huidobro (editores). 2015. Mercado escolar y oportunidad educacional en Chile: Libertad, diversidad y desigualdad. Santiago: Ediciones UC.

Couch, J. F., W. F. Shughart \& A. L. Williams. 1993. "Private School Enrollment and Public School Performance". Public Choice 76 (4): 301-312.

Drago, J. L. \& R. D. Paredes. 2011. "The Quality Gap in Chile's Education System". Cepal Review 104: 161-174.

Dronkers, J. \& S. Avram. 2015. "What Can International Comparisons Teach Us about School Choice and Non-Governmental Schools in Europe?". Comparative Education 51 (1): 118-132.

Elacqua, G. \& R. Fábrega. 2004. El consumidor de la educación: El actor olvidado de la libre elección de escuelas en Chile. Santiago de Chile: PREAL.

Gallego, F. A. 2002. "Competencia y resultados educativos: Teoría y evidencia para Chile". Cuadernos de Economía 39 (118): 309-352.

Gallego, F. A., A. E. Hernando, L. Flabbi \& M. Tartari. 2008. "On the Determinants and Implications of School Choice: Semi-Structural Simulations for Chile" [with comments]. Economía 9 (1): 197-244.

Gallego, F. \& C. Sapelli. 2007. "Financiamiento y selección en educación: Algunas reflexiones y propuestas". Puntos de Referencia 286, Centro de Estudios Públicos.

Gallegos, J., R. Chumacero \& R. Paredes. 2016. "Competition Pressures and Academic Performance in a Generalized Voucher Context". Estudios de Economía 43 (2): 217-232.

Gómez, D., R. A. Chumacero \& R. D. Paredes. 2012. "School Choice and Information". Estudios de Economía 39 (2): 143-157.

Grau, N., D. Hojman \& A. Mizala. 2015. "Destructive Creation: School Turnover and Educational Attainment". Serie Documentos de Trabajo 396, Facultad de Economía y Negocios, Universidad de Chile.

Hansen, B. E. 2000. "Sample Splitting and Threshold Estimation". Econometrica 68 (3): 575-603.

Hastings, J. S. \& J. M. Weinstein. 2008. "Information, School Choice, and Academic Achievement: Evidence from Two Experiments". The Quarterly Journal of Economics 123 (4): 1373-1414.

Hastings, J. S., R. van Weelden \& J. Weinstein. 2007. "Preferences, Information, and Parental Choice Behavior in Public School Choice". Working Paper 12995, National Bureau of Economic Research.

Hoxby, C. M. 1994. "Do Private Schools Provide Competition for Public Schools?" Working Paper 4978, National Bureau of Economic Research. 
2001. "How School Choice Affects the Achievement of Public School Students". En Choice with Equity, editado por P. T. Hill, 141-178. Stanford, CA: Hoover Institution Press.

Hsieh, C. T. \& M. Urquiola. 2003. "When Schools Compete, How Do They Compete? An Assessment of Chile's Nationwide School Voucher Program”. Working Paper 10008, National Bureau of Economic Research.

Kisida, B. \& P. J. Wolf. 2010. "School Governance and Information: Does Choice Lead to Better-Informed Parents?" American Politics Research 38 (5): 783805.

Ladd, H. F. \& E. B. Fiske. 2001. "The Uneven Playing Field of School Choice: Evidence from New Zealand". Journal of Policy Analysis and Management 20 (1): 43-64.

Levin, H. M. 1998. "Educational Vouchers: Effectiveness, Choice, and Costs". Journal of Policy Analysis and Management 17 (3): 373-392.

McEwan, P. J. \& M. Carnoy. 2000. "The Effectiveness and Efficiency of Private Schools in Chile's Voucher System". Educational Evaluation and Policy Analysis 22 (3): 213-239.

Neal, D. 2002. "How Vouchers Could Change the Market for Education". Journal of Economic Perspectives 16 (4): 25-44.

Pandey, L., D. L. Sjoquist \& M. B. Walker. 2009. “An Analysis of Private School Closings". Education 4 (1): 34-59.

Paredes, R., M. Opazo, P. Volante \& J. R. Zubizarreta. 2013. "Financiamiento compartido en la educación subvencionada chilena". En Propuestas para Chile. 51-84. Santiago: Centro de Políticas Públicas UC.

Paredes, R. D. \& J. I. Pinto. 2009. “¿El fin de la educación pública en Chile?” Estudios de Economía 36 (1): 47-66.

Phipps, A. G. 1993. “An Institutional Analysis of School Closures in Saskatoon and Windsor". Environment and Planning A 25 (11): 1607-1626.

Sander, W. 1999. "Private Schools and Public School Achievement". Journal of Human Resources 34 (4): 697-709.

Wongsosaputro, J., L. Pauwels \& F. Chan. 2011. "Testing for Structural Breaks in Discrete Choice Models". En Proceedings of 19th International Congress of MODSIM, 1652-1658. Modeling and Simulation Society of Australia and New Zealand Inc.

Zubizarreta, J. R., R. D. Paredes \& P. R. Rosenbaum. 2014. "Matching for Balance, Pairing for Heterogeneity in an Observational Study of the Effectiveness of For-Profit and Not-For-Profit High Schools in Chile". The Annals of Applied Statistics 8 (1): 204-231. 


\section{ANEXO}

Tabla A1. CANTIDAD DE ESCUELAS QUE ABRIERON SEGÚN TIPO DE DEPENDENCIA

\begin{tabular}{|c|c|c|c|c|c|c|c|}
\hline \multirow[b]{3}{*}{ Año de apertura } & \multicolumn{6}{|c|}{ Tipo de dependencia } & \multirow[b]{3}{*}{ Tota } \\
\hline & \multicolumn{2}{|c|}{ Municipal } & \multicolumn{2}{|c|}{$\begin{array}{c}\text { Particular } \\
\text { subvencionado }\end{array}$} & \multicolumn{2}{|c|}{$\begin{array}{l}\text { Particular } \\
\text { pagado }\end{array}$} & \\
\hline & Entradas & $\%$ & Entradas & $\%$ & Entradas & $\%$ & \\
\hline 2002 & & & & & & & \\
\hline 2003 & 56 & 0,91 & 447 & 0,76 & 44 & 4,73 & 547 \\
\hline 2004 & 29 & 0,48 & 229 & 5,28 & 16 & 1,86 & 274 \\
\hline 2005 & 48 & 0,79 & 240 & 5,11 & 15 & 1,97 & 303 \\
\hline 2006 & 16 & 0,27 & 241 & 4,86 & 11 & 1,50 & 268 \\
\hline 2007 & 12 & 0,20 & 216 & 4,22 & 14 & 1,92 & 242 \\
\hline 2008 & 10 & 0,17 & 236 & 4,43 & 12 & 1,65 & 258 \\
\hline 2009 & 23 & 0,40 & 310 & 5,52 & 12 & 1,79 & 345 \\
\hline 2010 & 7 & 0,12 & 173 & 3,01 & 6 & 0,89 & 186 \\
\hline 2011 & 12 & 0,22 & 151 & 2,59 & 5 & 0,76 & 168 \\
\hline 2012 & 17 & 0,31 & 312 & 5,17 & 11 & 1,76 & 340 \\
\hline 2013 & 4 & 0,07 & 192 & 3,15 & 7 & 1,16 & 203 \\
\hline Total & 234 & & 2.747 & & 153 & & 3.134 \\
\hline
\end{tabular}

